\title{
Transcriptome sequencing and analysis of major genes involved in calcium signaling pathways in pear plants (Pyrus calleryana Decne.)
}

\author{
Yuanyuan $\mathrm{Xu}^{\dagger}$, Xiaogang $\mathrm{Li}^{\dagger}$, Jing Lin ${ }^{*}$, Zhonghua Wang, Qingsong Yang and Youhong Chang*
}

\begin{abstract}
Background: Pears (Pyrus spp. L.) are an important genus of trees that produce one of the world's oldest fruit crops. Salinity stress is a common limiting factor for plant productivity that significantly affects the flavor and nutritional quality of pear fruits. Much research has shown that calcium signaling pathways, mediated by Calcineurin B-like proteins (CBLS) and their interacting kinases (CIPKs), are closely associated with responses to stresses, including salt. However, little is known about the molecular mechanisms that govern the relationship between salt stress and calcium signaling pathways in pear plants. The available genomic information for pears has promoted much functional genomic analysis and molecular breeding of the genus. This provided an ample foundation for characterizing the transcriptome of pear under salt stress.

Results: A high-throughput Illumina RNA-seq technology was used to identify a total of 78,695 unigenes that were successfully annotated by BLASTX analysis, using the publicly available protein database. Additionally, 2,855 novel transcripts, 218,167 SNPs, 23,248 indels and 18,322 alternative splicing events occurred. Assembled unique sequences were annotated and classified with Gene Ontology (GO), Clusters of Orthologous Group (COG) and Kyoto Encyclopedia of Genes and Genomes (KEGG) analysis, which revealed that the main activated genes in pear are predominately involved in functions such as basic physiological processes, metabolic pathways, operation of cellular components, signal transduction mechanisms, and other molecular activities. Through targeted searches of the annotations, the majority of the genes involved in calcium signaling pathways were identified, among which, four genes were validated by molecular cloning, while 11 were validated by RT-qPCR expression profiles under salt stress treatment.

Conclusions: These results facilitate a better understanding of the molecular genetics and functional genomic mechanisms of salt stress in pear plants. Furthermore, they provide a valuable foundation for additional research on the molecular biology and functional genomics of pear and related species.
\end{abstract}

Keywords: Calcium signaling pathways, Pear (Pyrus calleryana Decne.), RNA-Seq, Salt stress, Transcriptome

\section{Background}

Soil salinization is a severe problem encountered worldwide in agricultural production that causes abiotic stress to plants and lowers crop growth and yields, thus impacting food security [1]. The accumulation of sodium in soils ultimately leads to high concentrations of sodium ions $\left(\mathrm{Na}^{+}\right)$within plant cells [2]. Primary sources of salinity

\footnotetext{
* Correspondence: lj84390224@126.com; cyh@jaas.ac.cn

${ }^{\dagger}$ Equal contributors

Jiangsu Academy of Agricultural Sciences; Jiangsu Key Laboratory for
Horticultural Crop Genetic Improvement, Institute of Horticulture, Nanjing

Jiangsu Academy of Agricultural Sciences; Jiangsu Key Laboratory for
Horticultural Crop Genetic Improvement, Institute of Horticulture, Nanjing 210014, People's Republic of China
}

(c) 2015 Xu et al. Open Access This article is distributed under the terms of the Creative Commons Attribution 4.0 International License (http://creativecommons.org/licenses/by/4.0/), which permits unrestricted use, distribution, and reproduction in any medium, provided you give appropriate credit to the original author(s) and the source, provide a link to the Creative Commons license, and indicate if changes were made. The Creative Commons Public Domain Dedication waiver (http://creativecommons.org/publicdomain/zero/1.0/) applies to the data made available in this article, unless otherwise stated.

include natural processes such as mineral weathering or fluctuation of sea water levels, along with anthropogenic activities such as irrigation [3]. An overload of salinity in plants is known to cause a series of negative effects including reduction in water availability, mineral imbalances, and ultimately altered seed germination and plant metabolism [4]. Given the world's accelerating population growth and corresponding pressure on food supplies, this serious problem demands global solutions, such as improvement of the salt tolerance of crops [5]. Thus, elucidating the molecular basis of saltstress signal transduction pathways and salt tolerance 
mechanisms is fundamental to understanding the biology of salt-tolerant plants, in order to support strategies for the design, genetic engineering and breeding of salttolerant crops [6].

Plants have evolved complex signaling pathways in response to salt-stress, and have acquired plasticity in metabolic functions and developmental switches to cope with such changing environmental conditions [6-8]. Plant responses to signals are encoded by different $\mathrm{Ca}^{2+}$ signatures. The major family of $\mathrm{Ca}^{2+}$ sensors includes calmodulins (CaMs), calcineurin B-like proteins (CBLs), calcium-dependent protein kinases (CDPKs/CPKs) and CBL interacting protein kinases (CIPKs). These sensors contribute to intricate signaling networks in plants, and they are encoded by complex families of genes $[9,10]$. Considerable recent attention has been directed at explaining the molecular basis of plant salt tolerance. Most studies have emphasized the significance of $\mathrm{Ca}^{2+}$ signals in reinstating cellular ion homeostasis, demonstrating the importance of the calcium sensor and signaling pathways involved in salt stress signal transduction such as the CBL-CIPK and CDPK pathways that were identified in Arabidopsis and rice [11-13]. Research has shown that different, interconnected pathways may jointly coordinate regulation of plant responses to salt stress $[6,8]$.

Pear trees (Pyrus spp. L.) are cultivated widely throughout the world. They are the third most important fruit crop in temperate zones, after grape and apple $[14,15]$. The genome of this genus in the family Rosaceae (subfamily Pomoideae) was published recently; it contains 42,812 genes, providing a rich resource for research by plant molecular biologists [15]. In recent years, the production of pears in Southeast coast of China is reportedly quite limited due to further spread of soil salinization [16]. Considerable effort has been directed at investigating salt stress in pear plants, especially its accumulation, translocation, and physiological and metabolic effects $[17,18]$. However, little is known about the mechanisms underlying the responses of pear plants to salt stress at the molecular level, especially regarding relationships among the key genes that control salt stress and calcium signaling pathways.

Following release of the pear genome sequence, several studies have reported functional genomics research based on the analysis of transcription-level data. For example, RNA-seq technology has been used to identify genes related to bud dormancy in P. pyrifolia, and to study transcriptome profiling of fruit development and maturation in $P$. bretschneideri 'Rehd' (Chinese white pear) [19-21]. Despite this obvious potential, to date, RNA-seq analyses of the genome-wide responses of major genes involved in calcium signaling pathways under salt stress have not been assessed in pears.
Our objective was to use the RNA-seq technology platform to characterize the transcriptome of pear seedlings that were subjected to salt stress. A large set of transcript sequences for pear was obtained to identify the majority of activated genes involved in this process. The candidate genes involved in calcium signaling pathways were identified successfully, and the sequence of representative genes and expression patterns were further validated. The leaves de novo transcriptome based on RNA-seq was comprehensively characterized in pear plants. This would provide a publicly available foundation of information to facilitate both an improved understanding of the molecular mechanisms that regulate calcium signaling pathways in plants under salt stress, and the genetic improvement of traits governing fruit quality in molecular breeding programs for pear plants.

\section{Results and discussion}

\section{Annotation of predicted proteins}

Among 95,727 unique sequences that we identified, 78,695 of them (82.21\%) significantly matched a sequence in at least one of the five public databases that we consulted (Table 1). The rate of annotated unigenes was higher than the range of previous studies in other species (58 \% in safflower flowers and $58.01 \%$ in Chinese fir), indicating the relative integrity and conserved functions of the assembled transcripts sequences in pear $[22,23]$. The size-distribution of the predicted proteins and coding sequence (CDS) are shown in Additional file 1A and B, respectively. ESTScan was used to predict the coding regions of the remaining $17.79 \%$ of the unique sequences $(17,032)$ that did not match any in the databases. An additional 1,824 unique sequences $(1.91 \%)$ also showed orientation in the coding sequence (Additional file $1 \mathrm{C}$ and D). The absence of homologous sequences in public databases may indicate that novel genes are specifically expressed in pear leaves, or, it may be due to other biological or technical biases such as assembly parameters. Moreover, the reason why some cDNAs are non-coding or highly variable needs to be further explored [24, 25].

Table 1 Summary statistics of functional annotation for pear unigenes in public databases

\begin{tabular}{lll}
\hline Public protein database & No. of unigene hits & Percentage \\
\hline NR & 72,616 & 75.86 \\
NT & 70,691 & 73.85 \\
SwissProt & 41,255 & 43.10 \\
KEGG & 36,179 & 37.79 \\
COG & 16,915 & 17.67 \\
GO & 54,279 & 56.70 \\
ALL & 78,695 & 82.21 \\
\hline
\end{tabular}


The E-value distribution of the top hits in the NR database showed that $34.42 \%$ of the mapped sequences had strong homology with the E-value $<1.0 \mathrm{E}^{-45}$, whereas $65.57 \%$ of the homolog sequences ranged from $1.0 \mathrm{E}^{-5}$ to $1.0 \mathrm{E}^{-45}$ (Fig. 1a). The distribution of similarity values showed that $66.1 \%$ of the query sequences had a similarity of more than $80 \%$, while $33.8 \%$ of the hits had a similarity ranging from $17 \%$ to $80 \%$ (Fig. 1b). In terms of species distribution, the majority of the annotated sequences corresponded to known nucleotide sequences of plant species, with $73.4 \%$ of the sequences showing the highest homology to sequences from Amygdalus persica, followed by Fragaria vesca subsp. vesca (13.4\%), Malus domestica (3.7 \%) and Vitis vinifera (1.4\%) (Fig. 1c). The two species with the most BLAST hits belonged to the Rosaceae family, indicating that the sequences of the pear transcripts obtained in the present study were annotated properly [26].
Functional annotation and classification of the assembled unique sequences

GO assignments can provide standardized vocabulary for assigning functions of the uncharacterized sequences and hence were used to classify the functions of the predicted genes in pear [27]. Based on sequence homology, 54,279 sequences were categorized into 55 functional groups, including biological processes, cellular components, and molecular functions (Fig. 2). Within each of the three main categories of GO classification, the dominant subcategories were "cellular process" $(35,718)$ among biological processes, "cell" $(41,752)$ among cellular components, and "catalytic activity" $(27,825)$ among molecular functions. That is, the major GO classifications involved in the annotated sequences controlled basic biological regulation and metabolism. These findings were consistent with previous studies of transcriptome analysis in radish roots and sweet potato tuberous roots [24, 28]. COG classification was used to further evaluate the effectiveness of the

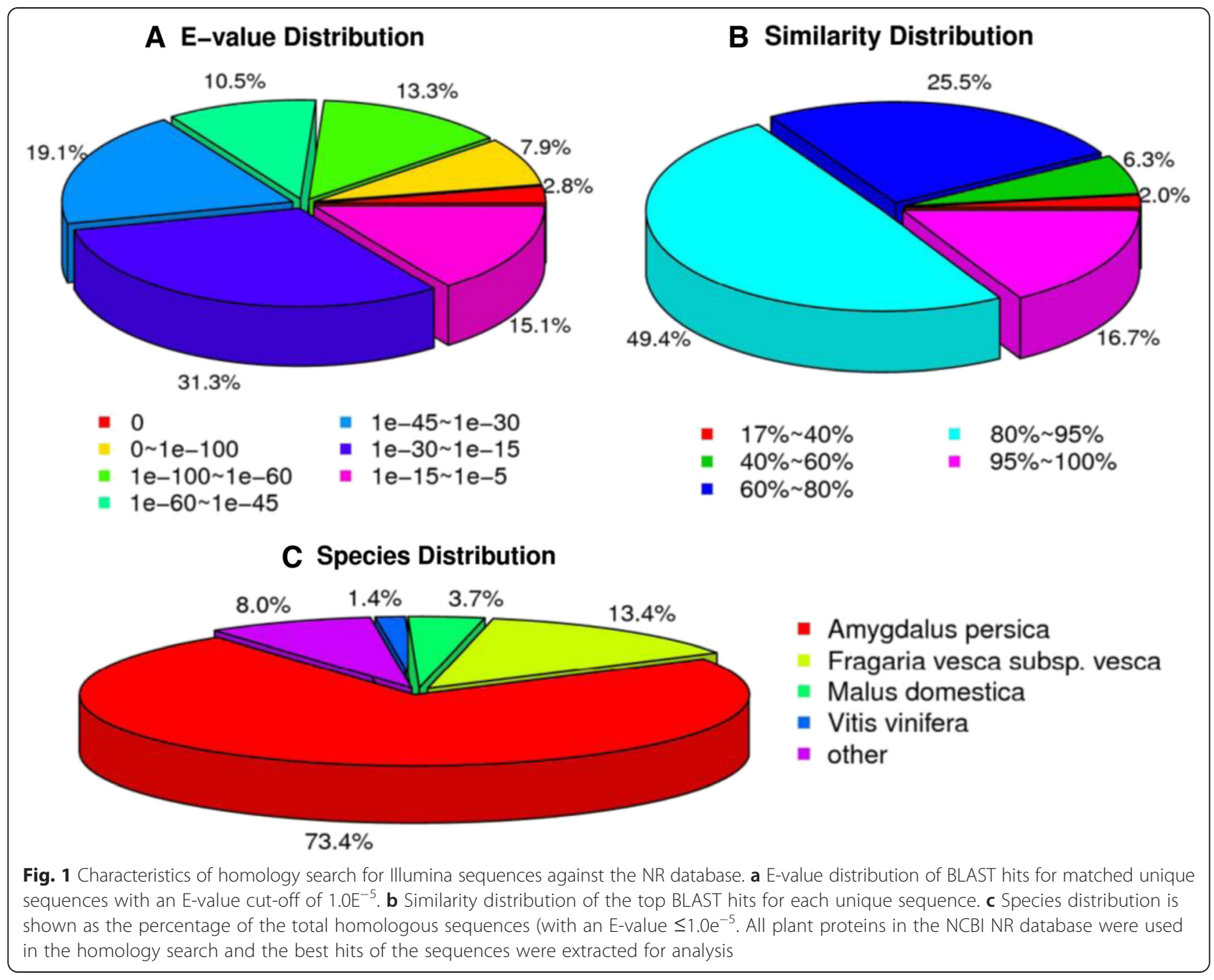




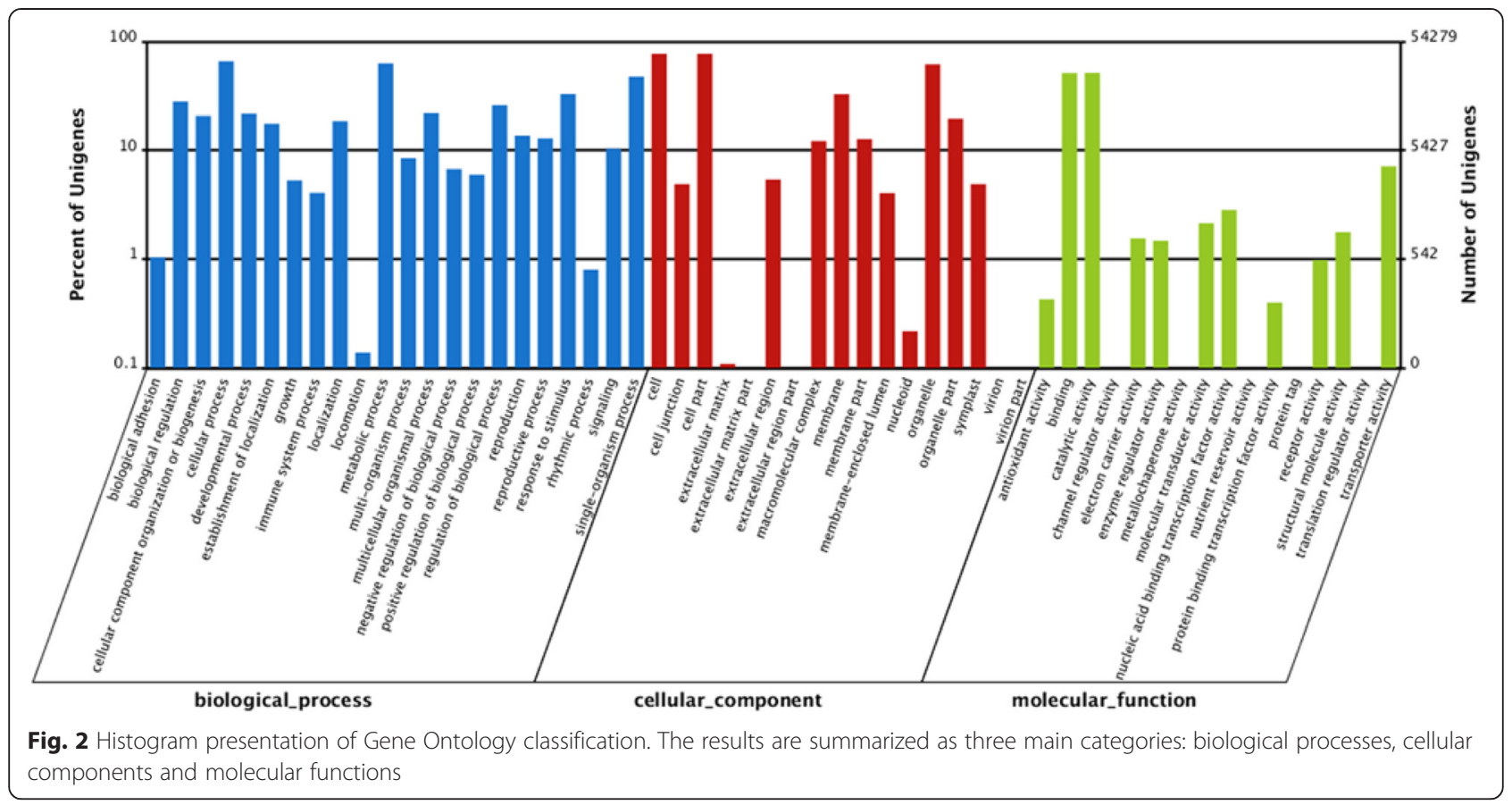

annotation process and the completeness of the transcriptome library. A COG database was built from classifications of phylogenetic relationships, consisting of protein sequences encoded in 21 complete genomes including those of bacteria, eukaryotes and algae [29]. Each COG classification consists of groups of paralogs or individual proteins from at least three lineages, and thus corresponds to an ancient conserved domain [25]. In total, 16,915 of 95,727 (17.67 \%) sequences were assigned to the COG classification (Table 1). As some of these sequences were annotated with multiple COG functions, altogether 29,337 functional annotations were generated. Among the 25 COG categories, the cluster for "General function prediction only" $(4,395,14.98 \%)$ represented the largest group, followed by "Transcription" (2,662, $9.07 \%)$, "Posttranslational modification, protein turnover, chaperones" (2,431, $8.29 \%)$ and "Replication, recombination and repair" $(2,381,8.12 \%)$. The categories of "extracellular structures" and "nuclear structures" were the smallest groups (Fig. 3).

The KEGG pathway database can facilitate a systematic understanding of the molecular interactions among genes, in terms of networks [30]. Pathway-based analysis enables further understanding of those biological functions. In order to identify the biological pathways active in pear, the unique sequences were annotated with KEGG Orthology (KO) numbers from BLASTX alignments against the KEGG database, using a cut-off $\mathrm{E}$ value of $10^{-5}$. A total of 36,179 (37.79\%) annotated sequences were matched in the KEGG database and were assigned to 128 active pathways. The five largest pathway groups were metabolic pathways [ko01100, 8435,
391 (23.31 \%)], biosynthesis of secondary metabolites [ko01110, 4,000 (11.06 \%)], plant-pathogen interactions [ko04626, 1,904 (5.26\%)], plant hormone signal transduction [ko04075, 1,814 (5.01 \%)] and spliceosomes [ko03040, 1,239 (3.42\%)] (Additional file 2). Figure 4 shows the features of the pathway assignment based on KEGG. The majority of the assigned unique sequences coded for related genes involved in primary metabolites, such as carbohydrate metabolism, lipid metabolism and amino acid metabolism. However, only 1,606 unique sequences that coded for the genes were associated with secondary metabolites. These annotations of protein or gene descriptions, putative conserved domains, and potential metabolic pathways will provide a valuable resource for subsequent investigation of specific processes, structures, biological functions and pathways in pear growth and development.

\section{Identification of novel transcripts, SNPs, indel and alternative splicing events}

During the RNA-Seq experiment, mRNA-enriched RNAs were firstly broken into short segments by chemical methods and then sequenced. Read alignment to the reference genome was performed using the TopHat/Cufflinks/ Bowtie/SOAP and Coding Potential Calculator [31]. Gene coverage was calculated as the percentage of a gene covered by reads. This value is equal to the ratio of the base number in a gene covered by unique mapping reads to the total number of bases in the coding region of that gene [32, 33]. A total of 78,023 (82\%) unique sequences with coverage between $90 \%-100 \%$ 


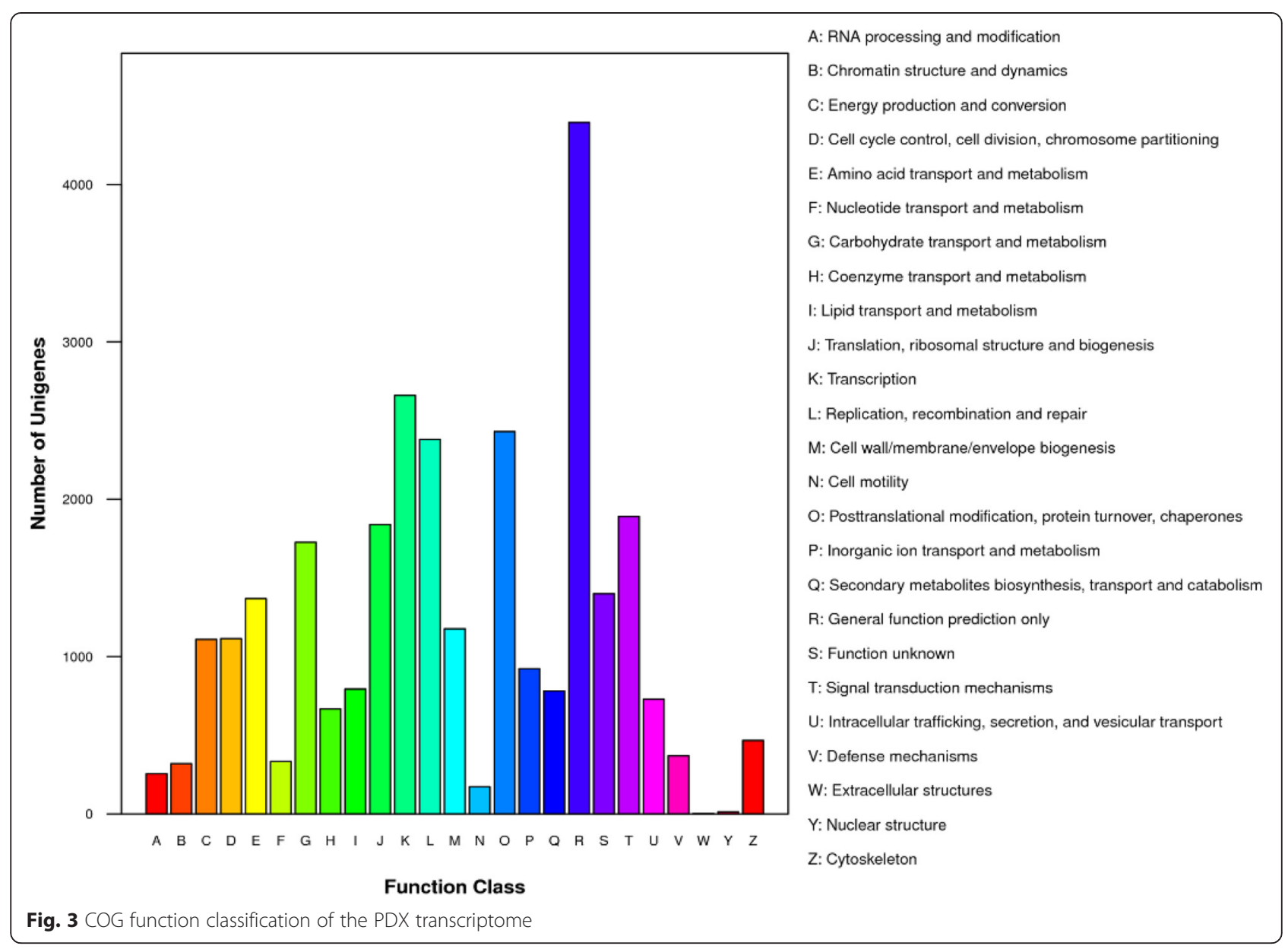

became the largest group, whereas no unique sequences were identified with coverage between 0 \%-20 \% (Fig. 5). After demultiplexing, merging and filtering of reads, about 26.8 million clean reads were obtained in the PDX sample of our experiment; $64.58 \%$ of the unique transcripts were sequenced and successfully mapped to the pear genome, and $45.39 \%$ were mapped to the de novo assembled results of pear (Table 2). As previously reported, an unprecedentedly high number of novel transcripts have been discovered in the genomes of humans, mice, and many plant species [31, 34]. In our research, a total of 2,855 transcripts were presumably displaying potentially novel isoforms for all the transcripts (Additional file 3). Additionally, results from Coding Potential Calculator analysis revealed that 1,720 potentially novel transcripts had the ability to code proteins, but the specific functions of these proteins remain to be determined (Fig. 6a). In order to provide the best genome coverage for the analysis of performance and production traits, numerous SNPs are needed $[35,36]$. SNPs include transition and transversion of the nucleotide; in this study, among the 218,167 SNPs found, $60.5 \%(132,070)$ were transitions between 'A/G' and 'C/T', whereas $39.5 \%(86,097)$ were transversions among ' $\mathrm{A} / \mathrm{C}, \mathrm{A} / \mathrm{T}, \mathrm{C} / \mathrm{G}$ and $\mathrm{G} / \mathrm{T}$ ' (Fig. 6b). The SNPs identified in this report provide a highly useful baseline for further genetic studies in pear, and will contribute to the development of a high density SNP array.

In addition, 23,248 insertion/deletion (indels) were identified, including 10,366 insertions and 12,881 deletions, with a homozygous:heterozygous ratio of 0.8 . Of the total, the proportion of single-base indels was highest $(8,545,36.8 \%)$, followed by double-base $(5,755,24.8 \%)$ and triple-base indels $(3,555,15.3 \%)$. The lengths of many indels were integral multiples of three bases, according to the indel length distribution (Fig. 6c). The indels were annotated according to the gene annotation information of birch-leaf pears. More than half of the indels were located in genic regions $(13,212,56.8 \%)$; another 10,036 were intergenic (43.2 \%). Among total 23,248 indels, 9,500 (40.8\%) were within regions $2 \mathrm{~kb}$ upstream or downstream of genes, while 536 indels were more than $2 \mathrm{~kb}$ away from genes. Furthermore, 6,110 (26.3 \%), 4,818 $(20.7 \%)$ and 2,269 (9.8 \%) indels among total 23,248 


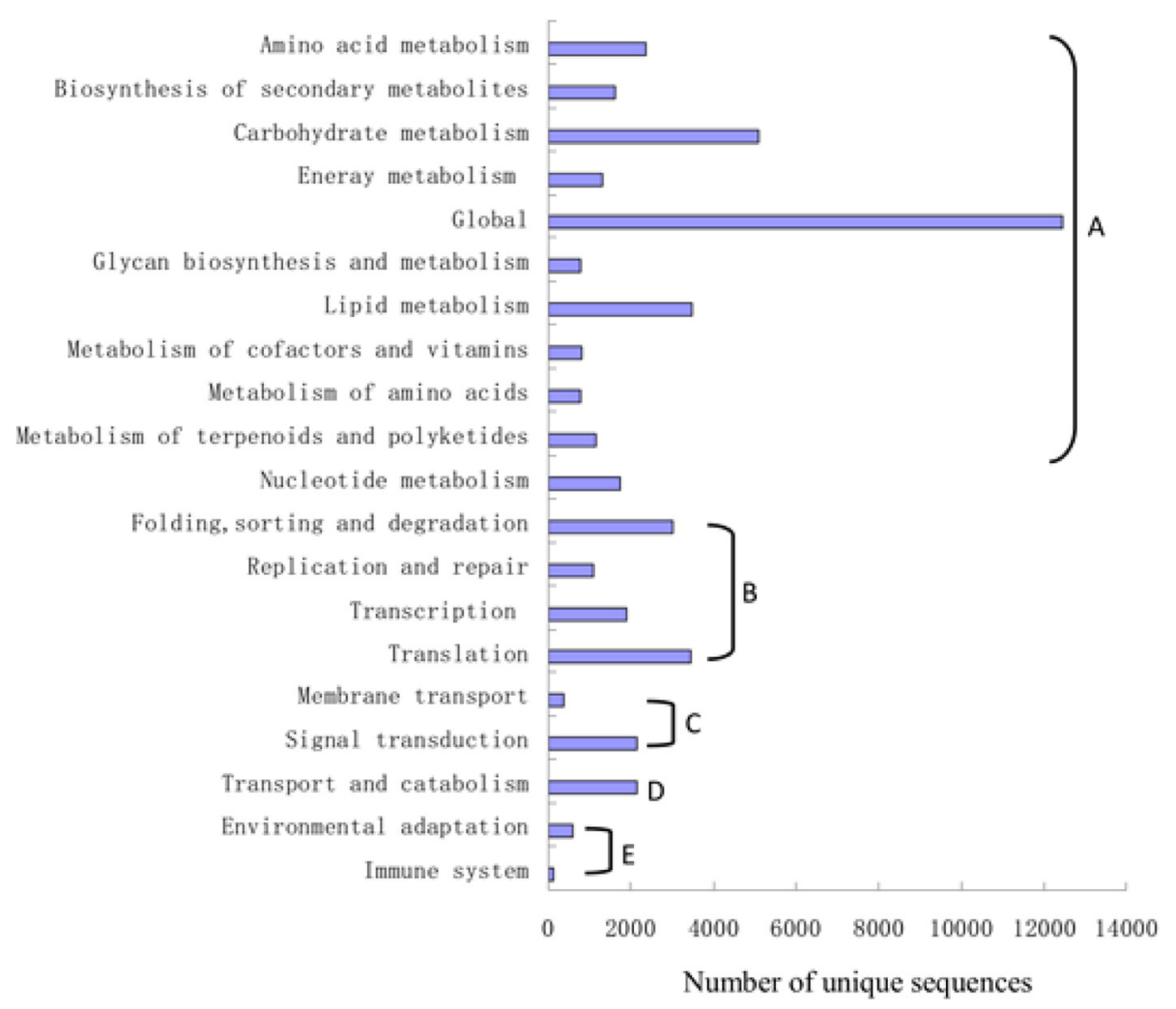

Fig. 4 Pathway assignment based on KEGG. Pathways were assigned into five categories. A: Metabolism; B: Genetic information processing; C: Environment information processing; D: Cellular processes; E: Organismal systems

indels were located within CDS, UTR and introns respectively, and 3,320 (14.3\%) indels led to frameshifts (Additional file 4).

The splicing of precursor mRNA not only reconstitutes gene coding sequences, but it is also an important regulatory step in the process of gene expression in plants [37, 38]. Alternative splicing is a major means of increasing the transcriptome and proteome diversity in plants or mammals for a given number of genes [39, 40]. RNA-seq provides an efficient opportunity to investigate

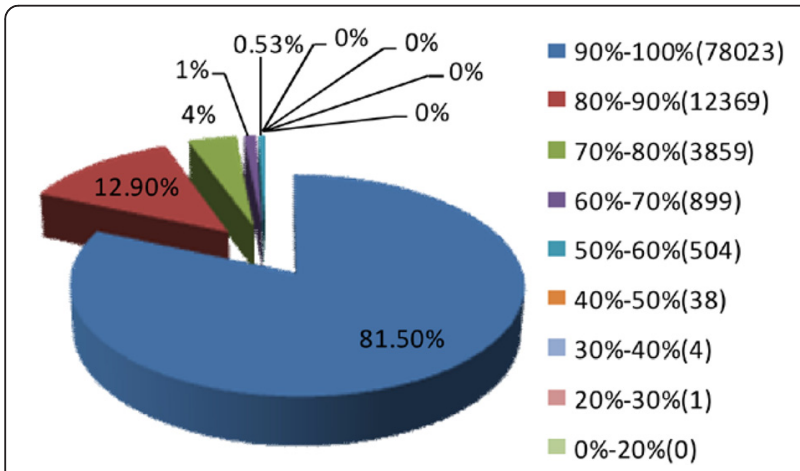

Fig. 5 Gene coverage distribution of PDX. Pie sections with different colors represent proportions of genes with certain coverage alternative splicing, which has revealed numerous instances of alternative splicing in mammals and plants [32, 41, 42]. We found evidence of 18,322 alternative splicing events, and IR was the major splicing pattern detected (67.8\% of total events), while ES was rare (only $1.9 \%$ of total events). The number of genes involved in the four types of alternative splicing were 316 for ES, 7,169 for IR, 2,339 for A5SS, and 2,621 for A3SS (Fig. 6d). These alignment results and analysis represented a significant improvement over the previous genome annotation and enabled us to further investigate gene expression profiles.

\section{Identification of genes involved in MYB transcription factors}

MYB transcription factors have been implicated in controlling cell development by responding to varieties of biotic and abiotic stresses, and by being involved in signal transduction processes of plant growth, including calcium signaling pathways [43, 44]. It has also been reported that promoters of related genes in calcium signaling pathways usually contain the same cis-acting elements, indicating that these promoters can be regulated by the same transcription factors [45]. The expression of the genes in calcium 
Table 2 Summary statistics for output sequencing

\begin{tabular}{llllllll}
\hline Sample name & Clean reads & Genome map rate & Gene map rate & Expressed genes & Novel transcripts & Alternative splicing events & SNPs \\
\hline PDX & $26,804,620$ & $64.58 \%$ & $45.39 \%$ & 95,697 & 2,855 & 18,322 & 218,167 \\
\hline
\end{tabular}

signaling pathways is regulated by the transcription regulation gene, thereby significantly improving the resistance of plants to salt stress. For example, MYB1 in Boea crassifolia, MYB4 in Oryza sativa and MYB2 in Chrysanthemum spp. showed a response to salt stress [46-48]. Soybean MYB transcription factors were also identified as being part of the stress tolerance apparatus based on salt stress assays in transgenic plants [49]. From the pear transcriptome analysis in this study, a total of 633 unique sequences were predicted to code MYB proteins including a large number of members (e.g., MYB1, 6, 15, 17, 32, 44, 58, 78, 107, 108, 109, 112, 113, 114, 115, 116, 118; Additional file 5). However, the specific function of each particular MYB member in calcium signaling pathways or salt-stress responses in pear needs to be verified using a functional genomics approach.
Identification of genes involved in the calcium signaling pathways of pear

Previous studies suggest that $\mathrm{Ca}^{2+}$ plays an important role in the signal transduction pathway of eukaryotes $[6,7]$. In plants, intracellular calcium levels are altered in response to various environmental stresses, including high concentrations of salts [8]. An additional level of regulation in calcium signaling is achieved via the action of calciumbinding proteins, including CDPKs, CaMs, CMLs and CBLs [9]. Based on the genes currently known to be involved in calcium signaling pathways in A. thaliana and rice, 75 unigenes in our transcriptome dataset were found to be homologous to the previously identified genes encoding calcium-binding proteins. The results indicated that those genes control responses to salt stress signals, and that the function is relatively conserved among different species. Moreover, four unigenes were found to

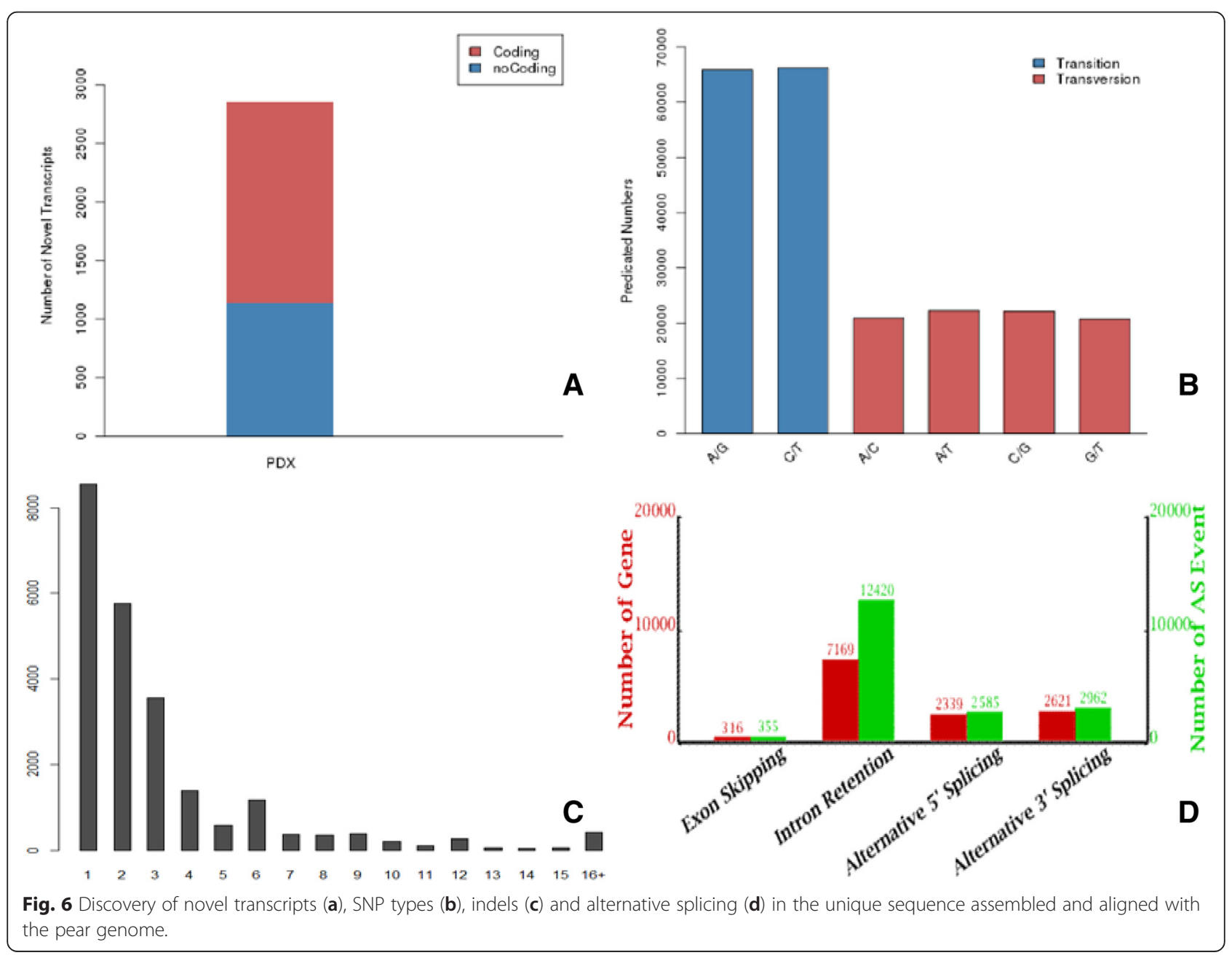


be homologous to the genes encoding calcineurin Blike protein (Additional file 6), which plays a critical role in responses to salt stress. In most cases, more than one unique sequence was annotated as encoding the same protein; such sequences may represent different members of a gene family and/or fragments of a single transcript [50].

CaMs play important roles in the regulation of growth, development and abiotic stress resistance and they have been identified in several plant species [51-53]. The $C M L$ family is likely to have evolved from CaMs. Genome-wide identification of $A$. thaliana and rice has revealed that $C a M$ and $C M L$ proteins are typically encoded by gene families. The A. thaliana genome harbors $7 \mathrm{CaM}$ and 50 $C M L$ genes that encode potential calcium sensors [51]. In our annotated pear transcriptome unigene dataset, 32 sequences were successfully identified that corresponded to seven homologous CaM genes (CaM 1-4 and 7-9) and 10 sequences that encoded calmodulin-like proteins (CMLs, K13448; Additional file 6).

CBL proteins fulfill crucial functions in diverse $\mathrm{Ca}^{2+}$ dependent processes in plants. CBL-CIPK complexes are instrumental in relaying plant responses to many environmental signals and in regulating ion fluxes [11-13, 54]. Genome analyses have revealed that 10 CBLs that specifically interact with distinct family members of the 26 CIPKs form a network-like signaling system for specific stimulus-response coupling [54, 55]. Our study finally discovered four unigene sequences that encode CBLs and 11 sequences that encode CIPKs (Additional file 6).

Among several known classes of $\mathrm{Ca}^{2+}$ binding sensory proteins, CDPKs are the best characterized and they are particularly important. Currently, most of the known calcium-stimulated protein kinase activities in plants are associated with CDPKs. Analysis of the genome sequence of Arabidopsis has indicated the presence of 34 CDPKs [56]. Based on sequence similarities, a total of 18 unigenes ranging from $151 \mathrm{bp}$ to $1536 \mathrm{bp}$ in the RNAseq dataset were identified as CDPKs, including CDPK1, 2, 5, 6, 9, 11, 19 and 23 (Additional file 6).

\section{Validation and expression analysis of genes involved in calcium-dependent signal transduction pathways}

Full-length cDNA sequences of four selected genes from $C B L s$ in $\mathrm{Ca}^{2+}$ dependent signal transduction pathways were isolated by T-A cloning, and compared with the unique sequences to check the quality of the annotation data from Solexa sequencing. The full cDNA length of these genes varied from $639 \mathrm{bp}$ to $801 \mathrm{bp}$ and the DNA length ranged from 1,788 bp to 2,969 bp. Overall, the assembled unique sequences covered more than $98 \%$ of the corresponding genome and the sequence variation was minimal ( $>98 \%$ pairwise identity), which confirmed that the RNA-seq based procedures of next generation sequencing were reliable (Table 3).

To further investigate the expression of genes involved in $\mathrm{Ca}^{2+}$-dependent signal transduction pathways, RTqPCR analysis was performed on eight selected genes, including four $C B L$ genes (PdCBL1, 2, 7 and 10) and six CDPK genes (PdCDPK1, 2, 5, 9, 10, 16 and 20), to provide detailed analysis of the effects of different concentrations of $\mathrm{NaCl}(0,100,200,300$ and $400 \mathrm{mM})$ after treatment for $6 \mathrm{~h}$. Several genes involved in calcium signaling pathways showed distinct patterns of expression in different species undergoing salt stress, such as $C B L$ genes in A. thaliana and Populus [57-59], and CDPK genes in A. thaliana, Oryza sativa, Triticum aestivum, Nicotiana tabacum and Glycine max [60-64]. The expression of all these genes in pear leaves varied with $\mathrm{NaCl}$ concentration (Fig. 7). Levels of PdCBL7 declined gradually as the strength of $\mathrm{NaCl}$ treatment increased. Expressions of the remaining genes were all up-regulated compared to the control, except that $P d C B L 2$ showed down-regulated in response to $\mathrm{NaCl}$ exposure for 100 and $200 \mathrm{mM}$ and $P d C B L 10$ was down-regulated at $400 \mathrm{mM}$.

\section{Conclusions}

In this study, NGS-based RNA sequencing for transcriptome methods (RNA-seq) was employed to characterize the responses of pear plants exposed to salt stress. A total of 78,695 unigenes were annotated successfully by BLASTX analysis using several publicly available protein databases. The main genes that were activated following the exposure of pear leaves to salt were predominately involved in basic biological processes, biosynthesis of secondary metabolites, signal transduction mechanisms, and other cellular components and molecular functions (based on their matches in the COG, GO and KEGG databases). Additionally, $64.58 \%$ of the unique transcripts were sequenced and successfully mapped to the pear genome, and $45.39 \%$ were mapped to the de novo assembled results of pear. Furthermore, a total of 2,855 novel transcripts, 218,167 SNPs and 23,248 indels were identified successfully, and 18,322 alternative splicing events occurred. These findings demonstrated that the RNA-seq based technology is a rapid and cost-effective method for novel gene discovery in pear plants. The

Table 3 Sequence analyses of the four pear genes involved in $\mathrm{Ca}^{2+}$-dependent signal transduction pathways

\begin{tabular}{lllll}
\hline Gene & Full-length CDNA & Full-length DNA & Coverage & ORF similarity \\
\hline PdCBL1 & 642 & 2,969 & $99.53 \%$ & $100 \%$ \\
PdCBL2 & 681 & 1,927 & $98.09 \%$ & $98.13 \%$ \\
PdCBL7 & 639 & 1,788 & $98.13 \%$ & $99.39 \%$ \\
PdCBL10 & 801 & 1,983 & $98.60 \%$ & $99.33 \%$ \\
\hline
\end{tabular}




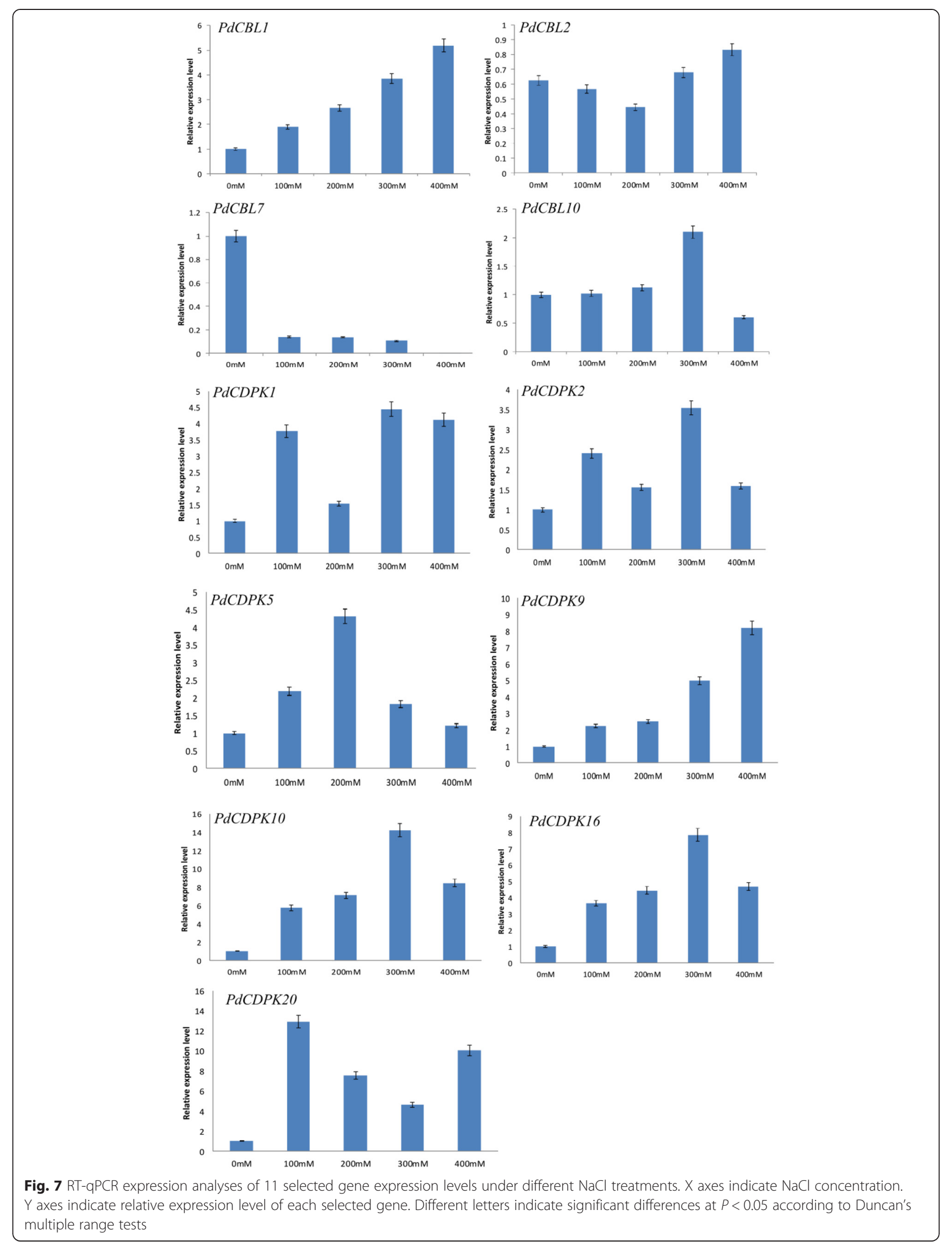


coverage of pear unigenes was comprehensive enough to allow for the discovery of almost all genes known to be involved in calcium signaling transduction and related pathways. The transcriptome dataset generated by this study will facilitate the understanding of molecular mechanisms via the regulation of calcium signaling. It will also provide experimental data useful for the development of salinity resistance in pear breeding programs.

\section{Methods}

\section{Plant materials and RNA extraction}

This study used seedlings of Callery Pear (P. calleryana 'Decne') growing at the National Pear Germplasm Repository in the Institute of Horticulture, Jiangsu Academy of Agricultural Sciences, Nanjing, China. Seeds that had been surface-sterilized and germinated were sown in a greenhouse maintained at $25^{\circ} \mathrm{C}$ for $14 \mathrm{~h}$ in light and $18{ }^{\circ} \mathrm{C}$ for $10 \mathrm{~h}$ in the dark. For transcriptome sequencing, seedlings at the 4-leaf stage were transferred from soil to $1 / 2 \mathrm{MS}$ solution (containing $200 \mathrm{mM} \mathrm{NaCl}$ ) and treated for $72 \mathrm{~h}$. Seedlings transferred to $1 / 2 \mathrm{MS}$ solution without $\mathrm{NaCl}$ were used as a control. Leaves were collected from the control and $\mathrm{NaCl}$ treated plants at the same time. For qRT-PCR verification, another set of seedlings at the 4-leaf stage was transferred to $1 / 2 \mathrm{MS}$ nutrient solution containing $0,100,200,300$ and $400 \mathrm{mM} \mathrm{NaCl}$, and the plants were exposed to their respective salt treatments for $6 \mathrm{~h}$. Leaves of all treated plants were collected at the same time. All samples were collected in triplicate, frozen in liquid nitrogen, and stored at $-80{ }^{\circ} \mathrm{C}$ until their use for total RNA extraction. Total RNA from the seedlings was isolated using the Spectrum Plant Total RNA Kit according to the manufacturer's protocol (Sigma-Aldrich, USA). RNase-free DNase I (Takara, Japan) was used to avoid DNA contamination. RNA samples were then quantified and examined for protein contamination (A260/A280) and reagent contamination (A260/A230) using a NanoDrop ND-1000 spectrophotometer.

\section{cDNA library preparation and Illumina sequencing}

Poly (A) mRNA was enriched from $20 \mu \mathrm{g}$ of total RNA from the $\mathrm{NaCl}$-treated pear samples, using Sera-mag Magnetic Oligo (dT) beads (Thermo Fisher Scientific, USA). To avoid priming bias when synthesizing cDNA, the purified mRNA was fragmented to small pieces ( 200 bp) using $1 \times$ fragmentation solution (Ambion, USA) at $94{ }^{\circ} \mathrm{C}$ for $5 \mathrm{~min}$. These short mRNA fragments were used as templates, and random hexamer-primers were used as primers for first-strand cDNA synthesis. Double-stranded cDNA was synthesized using the SuperScript Double-Stranded cDNA Synthesis Kit (Invitrogen, USA). After synthesis of the second strand of cDNA, cDNA fragments went through an end repair and
poly-A addition process, followed by ligation of sequencing adapters. The suitable fragment products were purified using agarose gel electrophoresis and enriched by PCR amplification to create a final library. The mixed cDNA library named 'PDX' was constructed using an mRNA-seq assay for paired-end transcriptome sequencing, which was performed by the Beijing Genomics Institute (Shenzhen, China) using an Illumina HiSeq ${ }^{\text {Tn }}$ 2000 platform, and 90 bp paired-end reads were generated. The sequencing data were deposited in the NCBI Sequence Read Archive (SRA, http://www.ncbi.nlm.nih.gov/sra/) as accession number SRX525736.

\section{Raw sequence processing and de novo assembly}

Raw reads generated by Illumina Hiseq ${ }^{\mathrm{TM}} 2000$ were initially processed to get clean reads. Then, de novo assembly of all clean reads was performed using the program Trinity (http://trinityrnaseq.github.io/). Firstly, clean reads with a certain length of overlap were combined to form longer contiguous sequences (contigs). The reads were then mapped back to contigs using Trinity to constract unigenes with the paired-end information. The distance and relation among these contigs was calculated based on paired-end reads, which enabled the detection of contigs from the same transcript and also calculation of distances among them. Next, the contigs were further connected with Trinity, and sequences that could not be extended on either end were obtained, and defined as unigenes [50].

\section{Functional annotation and classification of the unigene}

BLAST alignment was performed with a typical cut-off $E$ value of $10^{-5}$ between unigenes, using publically available protein data from several databases: NCBI non-redundant protein (NR), Swiss-Prot protein, Clusters of Orthologous Groups (COGs), Gene Ontology (GO), and the Kyoto Encyclopedia of Genes and Genomes (KEGG). The best aligning outputs were used to identify sequence direction of the unigenes. If results from different databases conflicted, we followed an order of priority of NR, Swiss-Prot, KEGG and COG. ESTScan was used to decide the sequence direction and to predict coding regions of a unigene if no alignment resulted from the above databases [65]. The BLAST2GO program with NR annotation was used to get GO annotations based on biological processes, molecular functions, and cellular components ontologies [66]. Then, WEGO software was used for GO functional classification of all unigenes, to understand the distribution of gene functions at a macro level [67].

\section{Detection of novel transcripts, alternative splicing, SNPs and indels}

To identify novel transcripts, assembled transcripts were compared to those for which reference sequences were annotated. Reference genome and gene model 
files were downloaded from the pear genome website at http://peargenome.njau.edu.cn/.

To be reported as a novel transcript, an assembled transcript must meet three requirements: it must be $\geq$ 200 bp away from the annotated gene, longer than $180 \mathrm{bp}$, and have a sequencing depth $\geq 2$. To discover the functions of the novel transcripts, the Coding Potential Calculator algorithm (http://cpc.cbi.pku.edu.cn/) was used to predict whether they had the ability to encode proteins $[31,68]$. We chose TopHat v1.4.0 to align paired-end clean reads to the reference genome, because this mapping tool can generate a database of splice junctions based on the gene model annotation file, thus producing a better mapping result than other non-splice mapping tools $[31,69]$. Clean reads were aligned to the reference genome using the Short Oligonucleotide Analysis Package (SOAP2), and then multi-mapped reads and duplicated reads were filtered from the alignment results in order to eliminate the PCR interference and ambiguous mapping $[32,70]$. Four basic types of alternative splicing were classified into exon skipping (ES), intron retention (IR), alternative 5 ' splicing site (A5SS) and alternative 3' splicing site (A3SS) [32, 71]. For intron retention, the retained regions needed to have at least ten-fold base coverage for each nucleotide position. Each accepted exon-exon junction had to be supported by at least two non-redundant reads spanning the junction. A single-nucleotide polymorphism (SNP) is a single nucleotide mutation that occurred in some individual samples based on DNA or RNA levels. SOAPsnp was used to detect SNPs; this program is a re-sequencing utility that can assemble a consensus sequence for the genome of a newly sequenced individual, based on alignment of raw sequencing reads with the known reference to obtain a consensus [70].

To detect indels, we used the Best Practices workflow for SNP and indel calling on RNAseq data available from the GATK website (https://www.broadinstitute.org/gatk/ guide/topic?name=methods\#methods3891). In brief, the reads were aligned to the genome of diploid $P$. bretschneideri Rehd. cv. Dangshansuli (also known as Suli) using STAR aligner. Specifically, we utilized the STAR 2-pass method, described recently [72]. Finally, the HaplotypeCaller was applied for indel calling. To ensure the accuracy of results, the results were filtered using stringent criteria (Fisher Strand values, FS $>200.0$, Qual By Depth values, QD $<2.0$, InbreedingCoeff $<-0.8$, and ReadPosRankSum $<-20.0)$, and the filtered results were annotated by SnpEff [73].

\section{Gene validation by T-A cloning and sequencing}

Degenerate and specific PCR primers were designed for the $C B L$ genes, corresponding to the conserved amino acid sequences in the analagous gene products of other plant species (Additional file 7). PCR was performed in a total volume of $25 \mu \mathrm{L}$ containing $2.0 \mathrm{mmol} / \mathrm{L} \mathrm{Mg}^{2+}$, $0.15 \mathrm{mmol} / \mathrm{L} \mathrm{dNTPs}, 0.4 \mathrm{mmol} / \mathrm{L}$ of each primer, $0.8 \mathrm{U}$ Taq DNA polymerase (TAKARA) and $10 \mathrm{ng}$ cDNA under the following conditions: an initial denaturation step at $94{ }^{\circ} \mathrm{C}$ for $3 \mathrm{~min}, 30$ cycles at $94{ }^{\circ} \mathrm{C}$ for $50 \mathrm{~s}, 58^{\circ} \mathrm{C}$ for $50 \mathrm{~s}, 72{ }^{\circ} \mathrm{C}$ for $100 \mathrm{~s}$, with a final extension at $72{ }^{\circ} \mathrm{C}$ for $10 \mathrm{~min}$ followed by holding at $4{ }^{\circ} \mathrm{C}$. The PCR products were separated by agarose gel electrophoresis and the incised gels were purified using the Agarose Gel DNA Purification Kit Ver.2.0 (TaKaRa). All products were ligated into the pMD19-T simple vector system (TaKaRa). Positive clones were sequenced with ABI3730 at Invitrogen Biotechnology Service Company (Shanghai, China).

\section{Quantitative real-time PCR analysis}

Quantitative real-time PCR was carried out in 96-well plates with an ABI7500 Real-Time PCR Detection System (Applied Biosystems, USA) using SYBR Green Master ROX (Roche, Japan) following the manufacturer's instructions. Primers were designed using Beacon Designer 7.0 (Premier Biosoft International, USA); Primer 5.0 (Premier Biosoft International, $\mathrm{CA}$ ) and the reference genes $A C T 2 /$ 7 and $U B Q 10$ were selected for use based on previous research (Additional file 8). Reaction mixtures contained $10 \mu \mathrm{L}$ SYBR Green Mix, $0.2 \mu \mathrm{M}$ of each primer and $1.5 \mu \mathrm{L}$ of 1:10 diluted cDNA as template in a final volume of $20 \mu \mathrm{L}$. Reactions were subjected to the following conditions: $95^{\circ} \mathrm{C}$ for $10 \mathrm{~min}$, followed by 40 cycles of $95^{\circ} \mathrm{C}$ for $30 \mathrm{~s}$ and $58{ }^{\circ} \mathrm{C}$ for $1 \mathrm{~min}[26,74]$. After the reactions, a melting curve analysis was conducted to evaluate the primer specificity. The relative expression levels of the selected transcripts, normalized to $A C T 2 / 7$ and $U B Q 10$, were calculated using the $2^{-\Delta \Delta \mathrm{Ct}}$ method. All reactions were performed with three independent biological replicates, and the expression levels calculated for each sample were based on three technical replicates. Data were analyzed using the ABI7500 manager software, and statistical analyses were conducted with SAS Version 9.0 software (SAS Institute, Cary, NC, USA) using Duncan's multiple range test at the $P<0.05$ level of significance.

\section{Additional files}

Additional file 1: Length distribution of the coding sequence (CDS) and predicted proteins by BLASTX and ESTScan software from the unique sequences. A: Aligned CDS by BLASTX. B: Proteins predicted by BLASTX. C: Aligned CDS by ESTScan. D: Proteins predicted by ESTScan. (DOC $2301 \mathrm{~kb}$ )

Additional file 2: KEGG pathways of the unique sequences. (XLS $712 \mathrm{~kb}$ )

Additional file 3: Identification and classification of novel transcripts based on the number of exons. (DOC $40 \mathrm{~kb}$ )

Additional file 4: Summary statistics for indel analysis. (DOC 39 kb) 


\section{Additional file 5: Candidate genes involved in MYB transcript} factors. (XLS $286 \mathrm{~kb}$ )

Additional file 6: Candidate genes involved in calcium signaling pathways in pear plants. (XLS $98 \mathrm{~kb}$ )

Additional file 7: Primers used for T-A cloning and sequencing. (DOC $33 \mathrm{~kb}$ )

Additional file 8: Primers used for RT-qPCR. (DOC $36 \mathrm{~kb}$ )

\section{Competing interests}

The authors declare that they have no competing interests.

\section{Authors' contributions}

$X Y, C Y$ and $L J$ designed the experiments and drafted the manuscript. $L X$ and $X Y$ planted pear seedlings, collected tissues and prepared the mRNA library for Solexa sequencing. WZ and YQ participated in the design of the study and performed the statistical analysis. $X Y, W Z$ and $Y Q$ participated in sequence alignment and gene validation and expression analysis. $C Y$ and $L J$ conceived of the study, participated in its design and coordination, and helped to draft the manuscript. All authors read and approved the final manuscript.

\section{Acknowledgements}

This work was supported by grants from the Program for the National Natural Science Foundation of China (Grant No. 31372051) and Post-doctoral Foundation of Jiangsu, China (Grant No.1302006B).

\section{Received: 25 July 2014 Accepted: 28 August 2015}

\section{Published online: 30 September 2015}

\section{References}

1. Parida AK, Das AB. Salt tolerance and salinity effects on plants: a review. Ecotoxicol Environ Saf. 2005:60:324-49.

2. Tuteja N. Mechanisms of high salinity tolerance in plants. Methods Enzymol. 2007:428:419-38

3. Munns R, Tester M. Mechanisms of salinity tolerance. Annu Rev Plant Biol. 2008:59:651-81.

4. Liu Y, Wang Q, Zhang Y, Cui J, Chen G, Xie B, et al. Synergistic and Antagonistic Effects of Salinity and $\mathrm{pH}$ on Germination in Switchgrass (Panicum virgatum L.). PLoS One. 2014;9:e85282.

5. Witzel K, Weidner A, Surabhi GK, Börner A, Mock HP. Salt stress-induced alterations in the root proteome of barley genotypes with contrasting response towards salinity. J Exp Bot. 2009;60:3545-57.

6. Guan Q, Wu J, Yue X, Zhang Y, Zhu J. A Nuclear calcium-sensing pathway is critical for gene regulation and salt stress tolerance in Arabidopsis. PLoS Genet. 2013;9:e1003755.

7. Ma S, Gong Q, Bohnert HJ. Dissecting salt stress pathways. J Exp Bot. 2005;57:1097-107.

8. Huang GT, Ma SL, Bai LP, Zhang L, Ma H, Jia P, et al. Signal transduction during cold, salt, and drought stresses in plants. Mol Biol Rep. 2012;39(2):969-87.

9. Dodd AN, Kudla J, Sanders D. The language of calcium signaling. Annu Rev Plant Biol. 2010;61:593-620

10. Batistič $\mathrm{O}$, Kudla J. Analysis of calcium signaling pathways in plants. Biochim Biophys Acta. 1820;2012:1283-93.

11. Kolukisaoglu U, Weinl S, Blazevic D, Batistic O, Kudla J. Calcium sensors and their interacting protein kinases: genomics of the Arabidopsis and rice CBL-CIPK signaling networks. Plant Physiol. 2004;134:43-58.

12. Luan S. The CBL-CIPK network in plant calcium signaling. Trends Plant Sci. 2009:14:37-42.

13. Weinl S, Kudla J. The CBL-CIPK Ca ${ }^{2+}$-decoding signaling network: function and perspectives. New Phytol. 2009;184:517-28.

14. Chang YH, Li H, Cong Y, Lin J, Sheng BL. Characterization and expression of a phytochelatin synthase gene in birch-leaf pear (Pyrus betulaefolia Bunge). Plant Mol Biol Rep. 2012;30:1329-37.

15. Wu J, Wang Z, Shi Z, Zhang S, Ming R, Zhu S, et al. The genome of the pear (Pyrus bretschneideri Rehd.). Genome Res. 2013;23(2):396-408.

16. WU QS, Zou YN. Adaptive responses of birch-leaved pear (Pyrus betulaefolia) seedlings to salinity stress. Not Bot Hort Agrobot Cluj. 2009:37:133-8.

17. Okubo M, Furukawa $Y$, Sakuratani T. Growth, flowering and leaf properties of pear cultivars grafted on two Asian pear rootstock seedling under $\mathrm{NaCl}$ irrigation. Sci Hortic. 2000;85:91-101.
18. Matsumoto K, Tamura F, Chun JP, Ikeda T, Imanishi K, Tanabe K. Enhancement in salt tolerance of Japanese pear by using Pyrus betulaefolia rootstock. Hortic Res (Japan). 2007:6:47-52.

19. Liu G, Li W, Zheng P, Xu T, Chen L, Liu D, et al. Transcriptomic analysis of 'Suli' pear (Pyrus pyrifolia white pear group) buds during the dormancy by RNA-seq. BMC Genomics. 2012;13:700.

20. Bai S, Saito T, Sakamoto D, Ito A, Fujii H, Moriguchi T. Transcriptome analysis of Japanese pear (Pyrus pyrifolia Nakai) flower buds transitioning through endodormancy. Plant Cell Physiol. 2013;54(7):1132-51.

21. Xie $M$, Huang $Y$, Zhang $Y$, Wang $X$, Yang $H$, Yu O, et al. Transcriptome profiling of fruit development and maturation in Chinese white pear (Pyrus bretschneideri Rehd). BMC Genomics. 2013;14:823.

22. Huang $L L$, Yang $X$, Sun $P$, Tong $W$, Hu SQ. The first Illumina-based de novo transcriptome sequencing and analysis of safflower flowers. PLOS One. 2012; $7: e 38653$

23. Huang HH, Xu LL, Tong ZK, Lin EP, Liu QP, Cheng $L$, et al. De novo characterization of the Chinese fir (Cunninghamia lanceolata) transcriptome and analysis of candidate genes involved in cellulose and lignin biosynthesis. BMC Genomics. 2012:13:648.

24. Wang Y, Xu L, Chen Y, Shen H, Gong Y, Limera C, Liu L. Transcriptome profiling of radish (Raphanus sativus L.) root and identification of genes involved in response to lead $(\mathrm{Pb})$ stress with next generation sequencing. Plos One. 2013b; 8:e66539.

25. Shi $C Y$, Yang $H$, Wei $C L$, Yu O, Zhang ZZ, Jiang CJ, et al. Deep sequencing of the Camellia sinensis transcriptome revealed candidate genes for major metabolic pathways of tea-specific compounds. BMC Genomics. 2011;12:131.

26. Dang ZH, Zheng LL, Wang J, Gao Z, Wu SB, Qi Z, et al. Transcriptomic profiling of the salt-stress response in the wild recretohalophyte Reaumuria trigyna. BMC Genomics. 2013;14:29.

27. Harris MA, Clark J, Ireland A, Lomax J, Ashburner M, Foulger R, et al. The Gene Ontology (GO) database and informatics resource. Nucleic Acids Res. 2004:32:258-61.

28. Xie F, Burklew CE, Yang Y, Liu M, Xiao P, Zhang B, et al. De novo sequencing and a comprehensive analysis of purple sweet potato (Impomoea batatas L.) transcriptome. Planta. 2012;236(1):101-13.

29. Dutkowski J, Tiuryn J. Identification of functional modules from conserved ancestral protein-protein interactions. Bioinformatics. 2007;23:i149-58.

30. Kanehisa M, Goto S. KEGG: kyoto encyclopedia of genes and genomes. Nucleic Acids Res. 2000;28:27-30.

31. Weikard R, Hadlich F, Kuehn C. Identification of novel transcripts and noncoding RNAs in bovine skin by deep next generation sequencing. BMC Genomics. 2013;14:789.

32. Zhang G, Guo G, Hu X, Zhang Y, Li Q, Li R, et al. Deep RNA sequencing at single base-pair resolution reveals high complexity of the rice transcriptome. Genome Res. 2010:20:646-54

33. Kang X, Liu G, Liu Y, Xu Q, Zhang M, Fang M. Transcriptome profile at different physiological stages reveals potential mode for curly fleece in Chinese tan sheep. PLoS One. 2013;8(8):e71763.

34. Harrow J, Frankish A, Gonzalez JM, Tapanari E, Diekhans M, Kokocinski F, et al. GENCODE: the reference human genome annotation for the ENCODE project. Genome Res. 2012:22(9):1760-74.

35. Li R, Li Y, Fang X, Yang H, Wang J, Kristiansen K, et al. SNP detection for massively parallel whole-genome resequencing. Genome Res. 2009;19(6):1124-32.

36. Canovas A, Rincon G, Islas-Trejo A, Wickramasinghe S, Medrano JF. SNP discovery in the bovine milk transcriptome using RNA-Seq technology. Mamm Genome. 2010;21:592-8.

37. Keren H, Lev-Maor G, Ast G. Alternative splicing and evolution: Diversification, exon definition and function. Nat Rev Genet. 2010:11:345-55.

38. Xiong J, Lu X, Zhou Z, Chang Y, Yuan D, Tian M, et al. Transcriptome analysis of the model protozoan, Tetrahymena thermophila, using deep RNA sequencing. PLoS One. 2012; 7:e30630.

39. Black DL. Mechanisms of alternative pre-messenger RNA splicing. Annu Rev Biochem. 2003;72:291-336.

40. Matlin AJ, Clark F, Smith CWJ. Understanding alternative splicing: towards a cellular code. Nat Rev Mol Cell Biol. 2005;6:386-98.

41. Filichkin SA, Priest HD, Givan SA, Shen RK, Bryant DW, Fox SE, et al. Genome-wide mapping of alternative splicing in Arabidopsis thaliana. Genome Res. 2010;20(1):45-58.

42. Wang ET, Sandberg R, Luo SJ, Khrebtukova I, Zhang L, Mayr C, et al. Alternative isoform regulation in human tissue transcriptomes. Nature. 2008:456:470-6. 
43. Jin H, Martin C. Multifunctionality and diversity within the plant MYB-gene family. Plant Mol Biol. 1999;41:577-85.

44. Dubos C, Stracke R, Grotewold E, Weisshaar B, Martin C, Lepiniec L. MYB transcription factors in Arabidopsis. Trends Plant Sci. 2010;15(10):573-81.

45. Yim H, Lee S, Lim M, Park M, Hwang Y. The promoter of CBL-interacting protein kinase 15 delivers the interference of sugar regulation by perturbed oxidative phosphorylation. Genes Genom. 2013;35:767-75.

46. Vannini C, Locatelli F, Bracale M, Magnani E, Marsoni M, Osnato M, et al. Overexpression of the rice Osmyb4 gene increases chilling and freezing tolerance of Arabidopsis thaliana plants. Plant J. 2004;37(1):115-27.

47. Chen BJ, Wang Y, Hu YL, Wu Q, Lin ZP. Cloning and characterization of a drought-inducible MYB gene from Boea crassifolia. Plant Sci. 2005;168:493-500.

48. Shan H, Chen S, Jiang J, Chen F, Chen Y, Gu C, et al. Heterologous expression of the chrysanthemum R2R3-MYB transcription factor CmMYB2 enhances drought and salinity, increases hypersensitivity to $A B A$ and delays flowering in Arabidopsis thaliana. Mol Biotechnol. 2012;51(2):160-73.

49. Liao Y, Zou HF, Wang HW, Zhang WK, Ma B, Zhang JS, et al. Soybean GmMYB76, GmMYB92, and GmMYB177 genes confer stress tolerance in transgenic Arabidopsis plants. Cell Res. 2008;18(10):1047-60.

50. Wang Y, Pan Y, Liu Z, Zhu X, Zhai L, Xu L, Yu R, Gong Y, Liu L. De novo transcriptome sequencing of radish (Raphanus sativus $L$.) and analysis of major genes involved in glucosinolate metabolism. BMC Genomics. 2013C; 14:836.

51. McCormack E, Tsai YC, Braam J. Handling calcium signaling: Arabidopsis CaMs and CMLs. Trends Plant Sci. 2005;10:383-9.

52. Parvin S, Lee OR, Sathiyaraj G, Khorolragchaa A, Kim YJ, Devi BS, et al. Interrelationship between calmodulin ( $\mathrm{CaM}$ ) and $\mathrm{H}_{2} \mathrm{O}_{2}$ in abscisic acidinduced antioxidant defense in the seedlings of Panax ginseng. Mol Biol Rep. 2012:39:7327-38.

53. Zhao Y, Liu W, Xu YP, Cao JY, Braam J, Cai XZ. Genome-wide identification and functional analyses of calmodulin genes in Solanaceous species. BMC Plant Biol. 2013;13:70.

54. Yu Q, An L, Li W. The CBL-CIPK network mediates different signaling pathways in plants. Plant Cell Rep. 2014;33:203-14.

55. Hashimoto K, Eckert C, Anschütz U, Scholz M, Held K, Waadt R, et al. Phosphorylation of Calcineurin B-like (CBL) calcium sensor proteins by their CBL-interacting protein kinases (CIPKs) is required for full activity of CBL-CIPK complexes toward their target proteins. J Biol Chem. 2012;287(11):7956-68.

56. Cheng SH, Willmann MR, Chen HC, Sheen J. Calcium signaling through protein kinases. The Arabidopsis calcium-dependent protein kinase gene family. Plant Physiol. 2002;129:469-85.

57. Shi J, Kim KN, Ritz O, Albrecht V, Gupta R, Harter K, et al. Novel protein kinases associated with calcineurin B-like calcium sensors in Arabidopsis. Plant Cell. 1999;11:2393-406.

58. Pandey GK, Cheong YH, Kim KN, Grant JJ, Li L, Hung W, et al. The calcium sensor calcineurin B-like 9 modulates abscisic acid sensitivity and biosynthesis in Arabidopsis. Plant Cell. 2004;16:1912-24.

59. Zhang H, Yin W, Xia X. Calcineurin B-like family in Populus: comparative genome analysis and expression pattern under cold, drought and salt stress treatment. Plant Growth Regul. 2008;56:129-40.

60. Urao T, Katagiri T, Mizoguchi T, Yamaguchi-Shinozaki K, Hayashida N, Shinozaki K. Two genes that encode $\mathrm{Ca}^{2+}$-dependent protein kinases are induced by drought and high-salt stresses in Arabidopsis thaliana. Mol Gen Genet. 1994;244(4):331-40.

61. Saijo Y, Hata S, Kyozuka J, Shimanoto K, Izui K. Over-expression of a single $\mathrm{Ca} 2+-$ dependent protein kinase confers both cold and salt/drought tolerance on rice plants. Plant J. 2000;23:319-27.

62. Zhang M, Liang S, Lu YT. Cloning and functional characterization of NtCPK4, a new tobacco calcium-dependent protein kinase. Biochim Biophys Acta. 2005;1729:174-85.

63. Liu F, Yoo BC, Lee JY, Pan W, Harmon AC. Calcium-regulated phosphorylation of soybean serine acetyltransferase in response to oxidative stress. J Biol Chem. 2006;281:27405-15.

64. Li AL, Zhu YF, Tan XM, Wang X, Wei B, Guo HZ, et al. Evolutionary and functional study of the CDPK gene family in wheat (Triticum aestivum L.) Plant Mol Biol. 2008;66(4):429-43.

65. Iseli C, Jongeneel CV, Bucher P. ESTScan: a program for detecting, evaluating, and reconstructing potential coding regions in EST sequences. Proc Int Conf Intell Syst Mol Biol. 1999:138-148.

66. Conesa A, Götz S, García-Gómez JM, Terol J, Talón M, Robles M. Blast2GO: a universal tool for annotation, visualization and analysis in functional genomics research. Bioinformatics. 2005;21(18):3674-6.
67. Ye J, Fang L, Zheng H, Zhang Y, Chen J, Zhang Z, et al. WEGO: a web tool for plotting GO annotations. Nucleic Acids Res. 2006;34:293-7.

68. Kong L, Zhang Y, Ye ZQ, Liu XQ, Zhao SQ, Wei L, et al. CPC: assess the protein-coding potential of transcripts using sequence features and support vector machine. Nucl Acids Res. 2007;35:W345-9.

69. Trapnell C, Roberts A, Goff L, Pertea G, Kim D, Kelley DR, et al. Differential gene and transcript expression analysis of RNA-seq experiments with TopHat and Cufflinks. Nat Protoc. 2012;7(3):562-78.

70. Li R, Li Y, Kristiansen K, Wang J. SOAP: Short oligonucleotide alignment program. Bioinformatics. 2008;24:713-4.

71. Zhou Y, Lu Y, Tian W. Epigenetic features are significantly associated with alternative splicing. BMC Genomics. 2012;13:123.

72. Engström PG, Steijger T, Sipos B, Grant GR, Kahles A, Consortium TR, et al. Systematic evaluation of spliced alignment programs for RNA-seq data. Nat Methods. 2013;10(12):1185-91.

73. Cingolani P, Patel VM, Coon M, Nguyen T, Land SJ, Ruden DM, et al. Using Drosophila melanogaster as a model for genotoxic chemical mutational studies with a new program SnpSift. Front Genet. 2012;3:35.

74. Xu Y, Zhu X, Gong Y, Xu L, Wang Y, Liu L. Evaluation of reference genes for gene expression studies in radish (Raphanus sativus L.) using quantitative real-time PCR. Biochem Biophys Res Commun. 2012;424:398-403.

\section{Submit your next manuscript to BioMed Central and take full advantage of:}

- Convenient online submission

- Thorough peer review

- No space constraints or color figure charges

- Immediate publication on acceptance

- Inclusion in PubMed, CAS, Scopus and Google Scholar

- Research which is freely available for redistribution 